\title{
Semi-stable production of bovine IL-4 and GM-CSF in the mammalian episomal expression system
}

\author{
Federico Carlos Blanco ${ }^{1,2^{*}}$, Cristina Lourdes Vazquez ${ }^{1,2 *}$ Julia Sabio y García ${ }^{1,2}$, \\ Rosana Valeria Rocha ${ }^{1,2}$, María José Gravisaco ${ }^{1,2}$, Marina Andrea Forrellad ${ }^{1,2}$, \\ Giovanni Magistrelli ${ }^{3}$, Fabiana Bigi ${ }^{1,2 \bowtie}$ \\ ${ }^{1}$ Institute of Agrobiotechnology and Molecular Biology, \\ National Institute of Agricultural Technology-National Scientific and Technical Research Council, \\ ${ }^{2}$ Institute of Biotechnology, Centre for Veterinary Scientific and Agronomics Research, \\ National Institute of Agricultural Technology, Nicolás Repetto y De los Reseros s/n, Hurlingham 1686, Buenos Aires, Argentina \\ ${ }^{3}$ Light Chain Bioscience - Novimmune SA, 1228 Plan-Les-Ouates Geneva, Switzerland \\ bigi.fabiana@inta.gob.ar
}

Received: January 22, $2021 \quad$ Accepted: July 26, 2021

\begin{abstract}
Introduction: Granulocyte-macrophage colony stimulating factor (GM-CSF) and interleukin-4 (IL-4) are cytokines widely used in ex vivo monocyte differentiation experiments, vaccine formulations and disease treatment. The aim of this study was to produce recombinant bovine GM-CSF and IL-4 in an episomal expression system that conserves the postransductional modification of the native proteins and to use the products to differentiate bovine monocytes into dendritic cells. Material and Methods: The recombinant proteins rGM-CSF and rIL-4 were expressed in PEAKrapid CRL-2828 human kidney cells, ATCC CRL-2828. The functional activity of the recombinant cytokines was monitored by registering morphological changes in bovine monocytes and assessing the expression of CD14 upon incubation with them. Results: Both recombinant proteins were detected in the cell culture supernatant of transfected cells. Culture supernatants of transfected cells induced in bovine monocytes morphological changes that resemble macrophages or dendritic cells. In addition, bovine cells treated with rGM-CSF and rIL-4 showed reduced expression of the macrophage surface marker CD14 compared with untreated cells. This effect indicates the expected differentiation. The expression of the cytokines was stable after many successive cell passages and a freeze/thaw cycle. Conclusion: The semi-stable mammalian episomal expression system used in this study allowed us to easily produce functional bovine rGM-CSF and rIL-4 without the need for protein purification steps.
\end{abstract}

Keywords: bovine GM-CSF, bovine IL-4, dendritic cells, macrophages, mammalian episomal expression.

\section{Introduction}

Dendritic cells (DCs) are unique antigen presenting cells with the ability to stimulate effective immune responses and develop/establish immune memory. They comprise a heterogeneous cell population that, according to ontogeny and function, includes conventional DCs (cDC1 and cDC2), plasmacytoid DCs (pDC) (28) and monocyte-derived DCs (MoDCs) (3).

Different functions are exercised by DCs, such as the regulation of $\mathrm{T}$-cell responses and the differentiation of T helper 1 (Th1), T helper 2 (Th2) or regulatory T (Treg) cells. Therefore, DCs play a central role in the immune responses against infectious and non-infectious diseases.
Researchers have deciphered numerous immune mechanisms from in vitro experimental evidence, in which primary cultures of cells from peripheral blood played a pivotal role. Primary DCs are difficult to isolate in sufficient quantities from tissue and, furthermore, do not proliferate in culture. In addition, DCs obtained directly from tissues often exhibit phenotypic heterogeneity. This is not the case with MoDCs, on the other hand, which are an excellent alternative, because monocytes are readily available in large quantities in peripheral blood and can easily be differentiated into DCs in vitro. Granulocyte macrophage colonystimulating factor (GM-CSF) and interleukin 4 (IL-4) are cytokines that facilitate the in vitro differentiation of 
adherent bovine monocytes from peripheral blood into MoDCs (2).

Macrophages are highly plastic cells that are traditionally divided into classical, or M1 (pro-inflammatory profile), and alternative, or M2 (anti-inflammatory profile) macrophages. Depending on the stimuli that human $\mathrm{M} 2$ receive, these cells polarise to four different phenotypes: M2a, M2b, M2c and M2d. Macrophages activated with IL-4 polarised to M2a, whereas those activated with GM-CSF polarised to M1 (18). In cattle, however, the role of GM-CSF and IL-4 in macrophage polarisation is still poorly understood.

To date, GM-CSF and IL-4 from different mammalian species have been expressed in many protein expression systems, including bacteria (29), yeast $(1,30)$, insect (14) and mammalian cells.

Different studies have reported the expression of bovine IL-4 as a protein secreted by Chinese hamster ovary cells (24) and in Trypanosoma brucei (8) as well as associated with Escherichia coli ghosts (11) and bovine GM-CSF expression has been successful in baculovirus/insect cells $(14,20)$ simian $\mathrm{CV}-1$ origin, SV40-carrying cells (17) and E. coli (16).

The aim of this study was to assess if the mammalian episomal expression system used allows efficient and stable expression of functional recombinant IL-4 and GM-CSF as well as the secretion of both cytokines to the cell culture supernatant.

\section{Material and Methods}

Construction of recombinant plasmids. Bovine GM-CSF and IL-4 gene sequences containing the Kozak motif at the 5 'end of each gene were amplified from plasmids kindly provided by Alejandra Capozzo (Instituto de Virología, CICVyA-INTA, Buenos Aires, Argentina) by using 5'gaattcTCAGTGATGGTGGTGAT3'/5' gaattc ACCATGTGGCTGCAGAAC3'and 5'aagettCTACTC TAGGGCACCTG3'/5' aagcttACCATGGGTCTCACC TACCAGCT3' primers. The amplified GM-CSF DNA product was cloned in the EcoRI site of the pEAK 8 vector, and the IL-4 amplicon was cloned in the HindIII site of the same vector. The pEAK 8 vector is an episomal replicative plasmid that confers resistance against puromycin to the transfected cells (19). Sequencing of the cloned genes showed that no mutations were introduced during the PCR amplification or cloning steps.

Cell transfection and protein production. PEAKrapid CRL-2828 human kidney cells (ATCC, Manassas, VA, USA) were seeded in 6-well cell culture plates at a density of $2 \times 10^{5}$ cells per well and incubated in Roswell Park Memorial Institute medium with foetal bovine serum (RPMI-FBS) at $37^{\circ} \mathrm{C}$ in $5 \% \mathrm{CO}_{2}$. The medium was removed on the following day and $80-90 \%$ confluent cell monolayers were transfected with $2 \mu \mathrm{g}$ of each recombinant plasmid plus $2 \mu \mathrm{g}$ of a green fluorescent protein plasmid and Lipofectamine 3000 reagent (Thermo Fisher Scientific, Waltham, MA, USA) following the manufacturer's recommendations. The following day, the transfection medium was removed and replaced with RPMI-FBS containing $1 \mu \mathrm{g} / \mathrm{mL}$ of puromycin (Sigma-Aldrich, St. Louis, MO, USA) for further incubation of the transfected cell monolayers at $37^{\circ} \mathrm{C}$ in $5 \% \mathrm{CO}_{2}$ for seven days. The cell transfection efficiency was estimated by observation of cell monolayers under an Eclipse TS100 microscope (Nikon Corporation, Tokyo, Japan) in epifluorescence mode. Transfected cells were detached from the wells and reseeded in T25 flasks. Then, cell cultures were amplified in T75 flasks in successive rounds in RPMIFBS medium. After seven days of culturing, culture supernatants were collected, filtered through a $0.22 \mathrm{~nm}$ pore membrane (Millipore, Billerica, MA, USA) and kept at $-80^{\circ} \mathrm{C}$.

Western blot. Transfected and non-transfected PEAKrapid CRL-2828 cells were cultivated in Ex-Cell 293 serum-free medium for human embryonic kidney (HeK) 293 cells (Sigma-Aldrich) supplemented with 1\% FSB and $1 \mu \mathrm{g} / \mathrm{mL}$ of puromycin (Sigma-Aldrich). Cell culture supernatants were harvested after 7-10 days and filtered through a $0.22 \mathrm{~nm}$ pore membrane (Millipore) and the filtered supernatants were incubated overnight at $4{ }^{\circ} \mathrm{C}$ in the presence of $10 \% \mathrm{v} / \mathrm{v}$ trichloroacetic acid (Sigma-Aldrich).

Total proteins were precipitated by centrifugation at $10,000 \mathrm{~g}$ for $30 \mathrm{~min}$ at $4^{\circ} \mathrm{C}$ and subsequently resuspended in a cracking buffer $(60 \mathrm{mM}$ Tris-Cl $\mathrm{pH} 6.8$, $2 \%$ sodium dodecyl sulphate (SDS), 10\% glycerol, $2 \% \beta$-mercaptoethanol, and $0.01 \%$ bromophenol blue) and to this suspension Trisma base $1 \mathrm{M}$ was added to neutralise the $\mathrm{pH}$ when necessary. Protein samples were heated at $100^{\circ} \mathrm{C}$ for $3 \mathrm{~min}$, subjected to electrophoresis in $15 \%$ sodium dodecyl sulphate-polyacrylamide electrophoresis (SDS-PAGE) gels, and then transferred to nitrocellulose membranes. The membranes were blocked with Tris-buffered saline (TBS) $(10 \mathrm{mM}$ Tris- $\mathrm{HCl}$ $\mathrm{pH} 7.5$, and $150 \mathrm{mM} \mathrm{NaCl}$ ) supplemented with 5\% skim milk for $30 \mathrm{~min}$ before incubation with primary antibodies (in-house polyclonal anti-bovine GM-CSF at 1: 100 or monoclonal anti-bovine IL-4 at 1: 50 (AbD Serotec, now Bio-Rad Antibodies, Hercules, CA, USA) for $2 \mathrm{~h}$. The nitrocellulose membranes were washed with TBS three times and incubated with a secondary antimouse alkaline phosphatase-conjugated antibody at a 1:10000 dilution for $2 \mathrm{~h}$. Western blots were revealed by incubation with 5-bromo-4-chloro-3'-indolyphosphate/ nitro blue tetrazolium (BCIP/NBT) solution (Promega, Madison, WI, USA).

Animals and sample collection. Healthy adult calves (36-60 months of age) were sampled from a herd settled in the National Institute of Agricultural Technology (INTA) with no history of diseases within the past two years. The animals were breed cross calves (Hereford and Aberdeen Angus). Blood samples (20 mL each) were collected via jugular vein with sterile disposable needles and syringes. Sample collection and animal handling were done in 
compliance with the regulations of the Ethical Committee of INTA (CICUAE).

Biological activity of the recombinant proteins. Heparinised blood $(20 \mathrm{~mL})$ from each animal was used for isolation of monocytes obtained from bovine peripheral blood mononuclear cells (PBMCs) by gradient centrifugation over Histopaque 1077 cell separation medium (Sigma-Aldrich) following the manufacturer's protocol. The mononuclear cells were kept frozen in liquid nitrogen until use, at which time they were thawed for $2 \mathrm{~min}$ in a $37^{\circ} \mathrm{C}$ bath, immediately resuspended in RPMI medium supplemented with $10 \%$ FBS, and centrifuged at $200 \mathrm{~g}$ for $7 \mathrm{~min}$. The cell pellets were resuspended in RPMI-FBS medium and seeded in 12- or 96-well cell culture plates. Non-adherent cells were removed after $4 \mathrm{~h}$ of incubation at $37^{\circ} \mathrm{C}$ in $5 \% \mathrm{CO}_{2}$ and the adherent cells were subsequently washed with phosphate-buffered saline (PBS) 1x. The adherent cells were incubated in RPMI-FBS supplemented with 1, 5, 10 or $20 \% \mathrm{v} / \mathrm{v}$ filtered culture supernatant of transfected cells for $1-7$ days at $37^{\circ} \mathrm{C}$ in $5 \% \mathrm{CO}_{2}$. Changes in bovine cell morphology were recorded under a TCS-SP5 optical/confocal microscope (Leica Microsystems GmbH, Wetzlar, Germany) using an HCX PL APO CS 40.0X 1.25 OIL UV 40× oil objective lens (Leica) and the $488 \mathrm{~nm}$ line of the argon laser for GFP excitation.

Flow cytometry. Each flow cytometry determination was performed using $2 \times 10^{6}$ cells from five different animals. The cells were stained with antiCD209 monoclonal antibody (cat. BOV2133, Washington State University Monoclonal Antibody Center, Pullman, WA, USA). Tubes with the corresponding isotype controls were set to assess nonspecific binding. Briefly, the cells were washed twice with PBS supplemented with 4\% FBS, labelled with anti-CD209 monoclonal antibody and, after another wash with PBS and FBS, stained with a secondary fluorochrome-conjugated antibody (Alexa Fluor 488-conjugated cat. no. ab150113, Abcam, Cambridge, UK). Finally, the cells were fixed with $0.5 \%$ paraformaldehyde (Sigma-Aldrich) and analysed in a FACScalibur cytometer (Becton Dickinson, Franklin Lakes, NJ, USA) using Cell Quest software (Becton Dickinson).

Real-time quantitative RT-PCR. Total RNA was extracted as previously described (15) from bovine adherent cells treated for 3 days with $10 \% \mathrm{v} / \mathrm{v}$ culture supernatant containing rGM-CSF and rIL-4 or culture supernatant of non-transfected cells. The synthesis of cDNA was performed using $1 \mu \mathrm{g}$ of DNA-free RNA, $50 \mathrm{ng}$ of random primers (Invitrogen, Thermo Fisher Scientific) and Moloney murine leukaemia virus reverse transcriptase (Promega). Each real-time qPCR reaction contained $25 \mathrm{ng}$ of cDNA as the template, Taq platinum (Invitrogen, Thermo Fisher Scientific), SYBR green I dye (Invitrogen, Thermo Fisher Scientific) and gene-specific primers (Supplementary Table 1). The reactions were performed in duplicate and in three biological replicates and run on a Step One Plus instrument (Applied
Biosystems, Thermo Fisher Scientific). Gene expression was analysed with LinReg software for curve analysis (26). Ratio calculation was performed with the Fg package of InfoStat statistical software (7). Differences in gene expression between the groups were analysed using gapd $H$ as the control gene.

\section{Results}

Production of semi-stable transfected cells expressing bovine rIL-4 and rGM-CSF. The bovine IL-4 and GM-CSF cDNA was cloned by RT-PCR from phorbol myristate acetate-activated PBMC. Interleukin 4 and GM-CSF cDNA with molecular weights of $411 \mathrm{bp}$ and $432 \mathrm{bp}$, respectively, was subcloned in the pEAK 8 vector, into which the Kozak sequence ACCATG was added upstream of the secretion signal sequences of the genes. The recombinant plasmids were used to transfect the PEAKrapid CRL-2828 cells. Together with the plasmid carrying the cytokine genes, a plasmid encoding $g f p$ was also transfected into the mammalian cells as a control of transfection. This reporter plasmid allowed us to estimate the success of the transfection, since it is assumed that in double transfections both plasmids form the complex with the lipid of the transfection reagent.

Both transfections displayed GFP+ cells after two days of culturing, as evidenced by optical microscopy under UV light (Fig. 1). This result indicates successful transfections, at least with the reporter plasmid.

\section{PEAKrapid ATCC $\bullet$ CRL-2828 ${ }^{\mathrm{m}}$ cells}

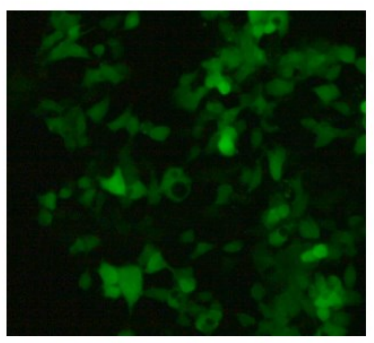

Cells transfected with pEAK 8 delete CMV I Promoter-IL-4+ plasmid expressing GFP

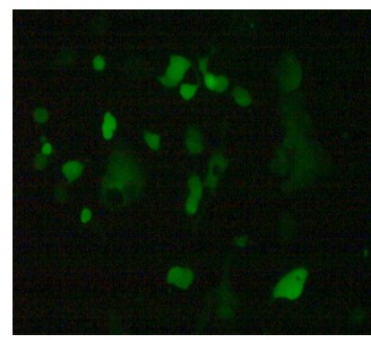

Cells transfected with pEAK 8 delete CMV I Promoter-GM-CSF+ plasmid expressing GFP
Fig. 1. PEAKrapid CRL-2828 cells transfected with plasmids expressing bovine IL-4 and bovine GM-CSF together with plasmids expressing GFP

In addition, transfected cells cultivated in the presence of puromycin in both Dulbecco's modified Eagle's medium and RPMI media supplemented with foetal bovine serum (FBS) showed no apparent differences in cell growth between the media (data not shown). For this reason, and because RPMI was more compatible with other experiments in this study, we subsequently selected this medium for use throughout the following experiments. The transfected cultures were expanded in medium containing puromycin in T75 flasks. 
To identify the recombinant cytokines in the culture supernatant of transfected cells, we then cultivated the cell monolayers in a synthetic medium supplemented with $1 \%$ FBS to avoid interference of bovine albumin in subsequent protein analyses. After five days of culturing, the culture supernatants were harvested and proteins were concentrated with $10 \%$ trichloroacetic acid. Total proteins were resolved in SDS-PAGE and transferred to nitrocellulose membranes. The recombinant rIL-4 and rGM-CSF were detected with a Western blot assay using specific antibodies. Polyclonal anti-bovine GM-CSF and monoclonal anti-bovine IL-4 recognised protein bands of $14-25 \mathrm{kDa}$ in culture supernatants of transfected cells; by contrast, no bands were observed under the nontransfected condition (Fig. 2). This result indicates that the transfected cells secreted bovine rIL-4 and rGM-CSF into the culture supernatant.

Biological activity of bovine rIL-4 and rGM-CSF . Subsequently, we evaluated the biological activity of rIL-4 and rGM-CSF in an ex vivo assay by incubating PBMCs with culture supernatant of transfected cells or non-transfected cells. Monocytes cultured with rIL-4 and rGM-CSF differentiated into MoDCs with a variety of morphological features, including the presence of many cells irregular in shape with long projections; monocytes cultured with culture supernatants of non-transfected cells, by contrast, did not display these morphological changes throughout the time of culture (Fig. 3).

Monocytes cultured in the presence of culture supernatant containing rGM-CSF alone displayed a cell morphology that shared some characteristics with those of bovine macrophages from a previous study (9)

(Fig. 3). However, this culture condition also presented cells with DC-like morphology (Fig. 3). Moreover, incubations of monocytes with rIL-4 alone showed spindle cells (Fig. 3).

After 60 days of culture, the transfected cells continued to secrete recombinant cytokines (data not shown). We evaluated the stability of transfected cells after freezing at $-80^{\circ} \mathrm{C}$ and thawing. Reseeding of frozen transfected cells after thawing produced recombinant cytokines with an equivalent biological activity to that of non-frozen cells (Fig. S1). Finally, lyophilisation and rehydration of culture supernatants apparently did not alter the integrity of either recombinant cytokine, as shown by the morphological changes induced in bovine monocytes incubated with rehydrated culture supernatant (Fig. S1).

$\alpha$ GM-CSF

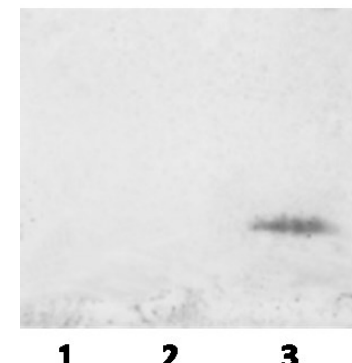

Fig. 2. Expression of rIL-4 and rGM-CSF in culture supernatants of transfected cells. Western blot analysis of total secreted proteins from transfected cells. Total proteins were precipitated with $10 \%$ TCA from culture supernatant of non-transfected cells (1) of cells transfected with plasmid expressing IL-4 (2 and 4) or GM-CSF (3and 5). Molecular weight marker was loaded on lane 6

$\alpha$ IL-4

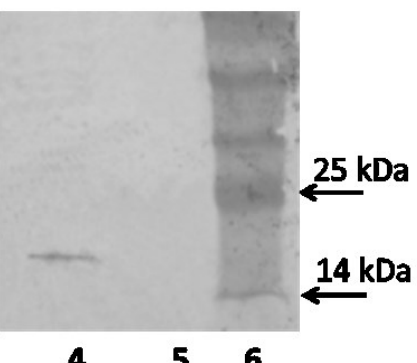

$25 \mathrm{kDa}$ $4 \mathrm{kDa}$

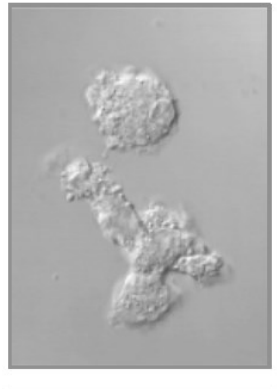

Control

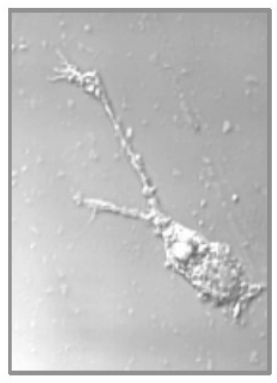

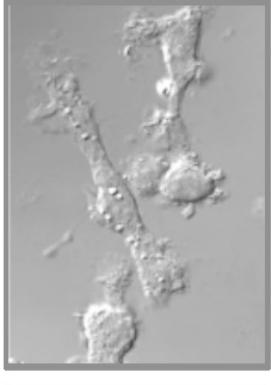

GM-CSF

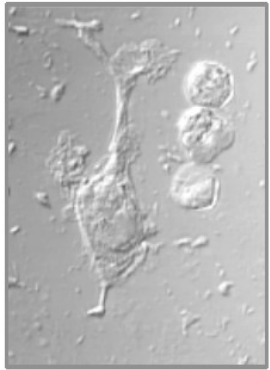

GM-CSF+IL-4

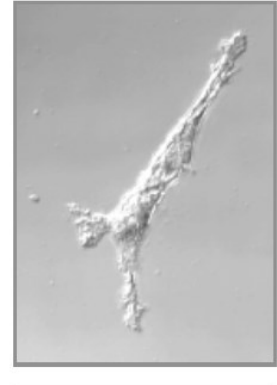

IL-4

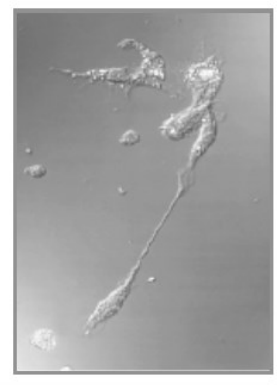

Ł

.

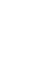


To determine the biological activity of rIL-4 and rGM-CSF, we evaluated the expression of CD209 in bovine monocytes incubated with the recombinant cytokines for $24 \mathrm{~h}$ or 5 days and using the commercial rIL-4/rGM-CSF mix and culture supernatant of nontransfected cells as positive and negative control conditions, respectively. Cells recovered from all culture conditions were CD209+ (\% CD209+ in negative control $=67$, in positive control $=49$, in rIL-4+GM-CSF treatment $=47$ ). The reason for these unexpected results is unclear; however, during the incubation time, monocytes may have maturated in vitro without the addition of any cytokine (M0). Therefore, despite the lack of significant changes in their morphology, nontreated cells expressed CD209.

As human monocytes highly express CD14, unlike MoDCs (3), we assessed the transcriptional level of the $c d 14$ gene in bovine monocytes (adherent cells) treated with culture supernatants of PEAKrapid cells expressing rIL-4 and rGM-CSF and the transcriptional level of this gene in untransfected cells. The adherent cells incubated for 3 days with rIl-4 and rGM-CSF showed significant reduction of $c d 14$ expression in comparison to the untreated cells (the fold change expression of untreated cells/cells treated with rIL-4rGM-CSF was 14.13, $\mathrm{SD}+/-4.49$, pval 0.023). This result confirmed that recombinant cytokines produced differentiation of bovine monocyte into DC.

\section{Discussion}

There are a wide variety of bacterial and eukaryotic expression systems available for the expression of recombinant cytokines. Bacterial systems have the advantage of being very profitable and easy to develop but they also have important drawbacks. Proteins are often insoluble and/or inactive. In addition, with some exceptions, bacteria do not glycosylate proteins and this lack of postransductional modification could be relevant for the biological activity of recombinant cytokines. Also, gram-negative bacteria such as E. coli produce potent immune response stimulators such as lipopolysaccharide and, therefore, additional purification steps are a prerequisite to eliminate any unwanted components.

Expression systems based on mammalian cells are best suited to correctly express functional cytokines, but their yields are frequently low. Finally, although baculovirus/insect cell expression systems are efficient in the expression of foreign proteins, the protein glycosylation pattern is different to that of mammalian cells.

In this study we expressed bovine IL-4 and GM-CSF in a semi-stable episomal mammalian cell system. The recombinant proteins were secreted to the culture supernatant without the addition of any protein tag. Therefore, the recombinant cytokines produced in this study are identical in their primary structures to those of the native proteins.
The cytokines produced here can be used not only for bovine DC studies or other immunological research topics, but also as powerful reagents for cattle disease treatments or vaccination strategies. For example, IL-4 has been used in foals as an adjuvant for an experimental vaccine against infection with equine herpesvirus type 1 (31). In addition, recombinant IL-4 in combination with other molecules has made the selection of transducted $\mathrm{T}$ cell for cancer therapies more efficient (25). On the other hand, recombinant GM-CSF has been widely used in cancer therapies, as reviewed by Parmiani et al. (23), and to prevent mastitis due to Staphylococcus aureus in cattle $(5,12,13,21)$.

One important limitation of the present study is that the recombinant proteins cannot be easily purified from the culture supernatant. This makes the estimation of the recombinant cytokine production extent impossible. However, we demonstrated that the culture supernatants of transfected cells induced morphological changes in bovine monocyte and that these changes resembled those of bovine dendritic cells or macrophages, thus indicating that functional rIL-4 and rGM-CSF were present in those culture supernatants. Moreover, the addition of only $1 \%$ of cell culture supernatants containing rIL-4/rGM-CSF to bovine monocytes induced the same morphological changes as $40-50 \mathrm{ng} / \mathrm{mL}$ of the commercial mix (Fig. S1). Another remarkable feature of this expression system is the feasibility of producing high volumes of supernatant containing recombinant cytokines in several successive cell passages. In this study we transfected a monolayer of about $2 \times 10^{6}$ cells and scaled the transfected cell cultures to $8.5 \times 10^{7}$, which allowed us to collect $200 \mathrm{~mL}$ of the culture supernatant containing cytokines. Moreover, the transfected cells maintained viability after storage at $-80^{\circ} \mathrm{C}$ and upon reseeding. These features make this semi-stable transfected cell technique a robust system to express recombinant proteins efficiently in their native forms.

Recently, Guo et al. (10) demonstrated that GM-CSF alone or in combination with IL-4 induces the maturation of monocytes to DCs. However, in the present experiment we found that the morphological changes induced by incubation with rGM-CSF alone in these cells are significantly different to those induced by incubation with both recombinant cytokines. Moreover, the treatment of bovine monocytes with culture supernatant containing rIL-4 also induced significant morphological changes. As mentioned above, human macrophages activated with IL-4 normally polarised to M2a, whereas those activated with GM-CSF polarised to M1. Rostam et al. (27) reported that human M2 macrophages are large, flattened cells, whereas M1 are elongated, spindle-shaped cells of smaller size. Remarkably, in our research, IL-4 macrophage polarisation resulted in cells resembling M1 human macrophages instead.

Unfortunately, there are no cell surface markers that can differentiate bovine DCs from bovine 
macrophages. Researchers have described CD209, together with CD205, as cell markers expressed in DCs; however, these receptors are also expressed by bovine macrophages (22). The current production established CD209 expression by bovine monocytes attached to the bottom well of culture plates treated with IL-4 or GM-CSF (or with both cytokines). This finding suggests that these treatments induced the differentiation of monocytes into macrophages or DCs. However, this C-type lectin receptor was also detected in the attached monocytes incubated in the absence of IL-4 or GM-CSF. This result is consistent with those of previous studies reporting that attachment triggers the differentiation of monocytes, called M0, and also supports other research demonstrating that FBS promotes monocyte maturation in vitro $(4,6)$. Therefore, the expression of CD209 was not suitable for differentiating the cell phenotypes induced by the recombinant cytokines. By contrast, the expression of $c d 14$ differentiated untreated monocytes (M0) from those treated with the recombinant cytokines. This finding supports the fact that functional rIL-4 and rGM-CSF secreted from transfected PEAKrapid cells induced polarisation of bovine monocytes to DCs with the concomitant loss of CD14 monocyte cell marker.

From the results of this study, we conclude that the recombinant cytokines expressed according to this method are biologically active. However, the cell shape changes observed after incubation of bovine monocytes with rIL-4 or rGM-CSF alone were not consistent with those reported for human cells. This finding suggests that the monocyte polarisation pathways are not completely conserved between humans and cattle.

* These authors contributed equally to this work.

Conflict of Interests Statement: The authors declare that there is no conflict of interests regarding the publication of this article.

Financial Disclosure Statement: This study was supported by INTA grants i102 and i105.

Animal Rights Statement: None required.

Acknowledgments. We thank Camila and Emilia Arzaroli and Fernando Bigi for their contribution of some reagents.

Supplementary material related to this article can be found in the online version.

\section{References}

1. Babu K.S., Pulicherla K.K., Antony A., Meenakshisundaram S.: Cloning and expression of recombinant human GMCSF from Pichia pastoris GS115-A progressive strategy for economic production. Am J Ther 2014, 462-469, doi: 10.1097/mjt.0000000 000000040 .

2. Chometon T.Q., da Silva Siqueira M., Sant Anna J.C., Almeida M.R., Gandini M., de Almeida Nogueira A.C.M., Zuquim Antas P.R.: A protocol for rapid monocyte isolation and generation of singular human monocytederived dendritic cells. PLoS One 2020, 15, doi: 10.1371/journal.pone.0231132.

3. Collin M., Bigley V.: Human dendritic cell subsets: an update. Immunology 2018, 154, 3-20, doi: 10.1111/imm.12888.

4. Czuprynski C.J., Hamilton H.: The effects of serum on the in vitro adherence and maturation of bovine monocytes. Vet Immunol Immunopathol 1985, 9, 189-193, doi: 10.1016/0165-2427(85) 90018-2.

5. Daley M., Williams T., Coyle P., Furda G., Dougherty R., Hayes P.: Prevention and treatment of staphylococcus aureus infections with recombinant cytokines. Cytokine 1993, 5, 276-284, doi 10.1016/1043-4666(93)90015-W.

6. de Mulder P.H., van Rennes H., Mier P.D., Bergers M., de Pauw B.E., Haanen C.: Characterization of monocyte maturation in adherent and suspension cultures and its application to study monocyte differentiation in Hodgkin's disease. Clin Exp Immunol 1983, 54, 681-688.

7. di Rienzo J.A., Casanoves F., Balzarini M.G., Gonzalez L., Tablada M., Robledo C.W.: InfoStat. Grupo Infostat, FCA, Universidad Nacional de Córdoba, 2014.

8. Furger A., Jungi T.W., Salomone J.Y., Weynants V., Roditi I.: Stable expression of biologically active recombinant bovine interleukin-4 in Trypanosoma brucei, FEBS Letters 2001, 508, 90-94, doi: 10.1016/S0014-5793(01)03031-9.

9. García-Sánchez M., Jiménez-Pelayo L., Horcajo P., Regidor-Cerrillo J., Ólafsson E.B., Bhandage A.K., Barragan A, Werling D., OrtegaMora L.M., Collantes-Fernández E.: Differential responses of bovine monocyte-derived macrophages to infection by Neospora caninum isolates of high and low virulence. Front Immunol 2019, 10, 915, doi: 10.3389/fimmu.2019.00915.

10. Guo X., Zhou Y., Wu T., Zhu X., Lai W., Wu L.: Generation of mouse and human dendritic cells in vitro. J Immunol Methods 2016, 432, 24-29, doi: 10.1016/j.jim.2016.02.011.

11. Hajam I.A., Dar P.A., Appavoo E., Kishore S., Bhanuprakash V., Ganesh K.: Bacterial ghosts of Escherichia coli drive efficient maturation of bovine monocyte-derived dendritic cells. PLoS ONE 2015,10, e0144397, doi: 10.1371/journal.pone.0144397.

12. Kehrli M.E., Cullor J.S., Nickerson S.C.: Immunobiology of Hematopoietic Colony-Stimulating Factors: Potential Application to Disease Prevention in the Bovine. J Dairy Sci 1991, 74, 4399-4412, doi: 10.3168/jds.S0022-0302(91)78636-0.

13. Kiku Y., Ozawa T., Takahashi H., Kushibiki S., Inumaru S., Shingu H., Nagasawa Y., Watanabe A., Hata E., Hayashi T.: Effect of intramammary infusion of recombinant bovine GM-CSF and IL- 8 on CMT score, somatic cell count, and milk mononuclear cell populations in Holstein cows with Staphylococcus aureus subclinical mastitis. Vet Res Commun 2017, 41, 175-182, doi: 10.1007/s11259-017-9684-y.

14. Kinoshita Y., Xu J., Masuda A., Minamihata K., Kamiya N., Mon H., Fujita R., Kusakabe T., Lee J.M.: Expression and purification of biologically active human granulocyte-macrophage colony stimulating factor (hGM-CSF) using silkworm-baculovirus expression vector system. Protein Expr Purif 2019, 159, 69-74, doi: 10.1016/j.pep.2019.03.010.

15. Klepp L.I., Eirin M.E., Garbaccio S., Soria M., Bigi F., Blanco F.C.: Identification of bovine tuberculosis biomarkers to detect tuberculin skin test and IFN $\gamma$ release assay false negative cattle. Res Vet Sci 2019, 122, 7-14, doi: 10.1016/j.rvsc.2018.10.016.

16. Leong S.R., Flaggs G.M., Lawman M.J.P., Gray P.W.: Cloning and expression of the cDNA for bovine granulocyte-macrophage colony-stimulating factor. Vet Immunol Immunopathol 1989, 21, 261-278, doi: 10.1016/0165-2427(89)90036-6.

17. Maliszewski C.R., Schoenborn M.A., Cerretti D.P., Wignall J.M., Picha K.S., Cosman D., Tushinski R.J., Gillis S., Baker P.E.: Bovine GM-CSF: Molecular cloning and biological activity of the recombinant protein. Mol Immunol 1988, 25, 843-850, doi: 10.1016/0161-5890(88)90120-4.

18. Mily A., Kalsum S., Loreti M.G., Rekha R.S., Muvva J.R., Lourda M., Brighenti S.: Polarization of M1 and M2 Human Monocyte-Derived Cells and Analysis with Flow Cytometry upon Mycobacterium tuberculosis Infection. J Vis Exp 2020, 163, doi: 10.3791/61807. 
19. Magistrelli G., Malinge P., Lissilaa R., Fagète S., Guilhot F., Moine V., Buatois V., Delneste Y., Kellenberger S., Gueneau F., Ravn U., Kosco-Vilbois M., Fischer N.: Rapid, simple and high yield production of recombinant proteins in mammalian cells using a versatile episomal system. Protein Expr Purif 2010, 72, 209-216. doi: 10.1016/j.pep.2010.04.007.

20. Nagaya H., Kanaya T., Tobita Y., Yokomizo Y., Inumaru S., Onodera T.: Development of efficient method for purified recombinant bovine granulocyte-macrophage colony-stimulating factor production with baculovirus-silkworm gene expression system. Biotechnol Lett 2008, 30, 41-45, doi: 10.1007/s10529007-9506-3.

21. Ozawa T., Kiku Y., Mizuno M., Inumaru S., Kushibiki S., Shingu H., Matsubara T., Takahashi H., Hayashi T.: Effect of intramammary infusion of rbGM-CSF on SCC and expression of polymorphonuclear neutrophil adhesion molecules in subclinical mastitis cows. Vet Res Commun 2012 36, 21-27, doi: 10.1007/s11259-011-9506-6.

22. Park K.T., ElNaggar M.M., Abdellrazeq G.S., Bannantine J.P., Mack V., Fry L.M., Davis W.C.: Phenotype and function of CD209+ bovine blood dendritic cells, monocyte-derived-dendritic cells and monocyte-derived macrophages. PLoS One 2016, 11, e0171059, doi: 10.1371/journal.pone.0165247.

23. Parmiani G., Castelli C., Pilla L., Santinami M., Colombo M.P., Rivoltini L.: Opposite immune functions of GM-CSF administered as vaccine adjuvant in cancer patients. Ann Oncol 2007, 18, 226-232, doi: 10.1093/annonc/mdl158.

24. Prashanth T., Reddy G.R., Suryanaryana V.V.S., Dechamma H.J.: Cloning and expression of Bos indicus interleukin-4 in mammalian cells. Indian J Exp Biol 2013, 51, 352-356.
25. Ptáčková P., Musil J., Štach M., Lesný P., Němečková Š., Král V., Fábry M., Otáhal P.: A new approach to CAR T-cell gene engineering and cultivation using piggyBac transposon in the presence of IL-4, IL-7 and IL-21. Cytotherapy 2018, 20, 507-520, doi: 10.1016/j.jcyt.2017.10.001

26. Ramakers C., Ruijter J.M., Deprez R.H., Moorman A.F.: Assumption-free analysis of quantitative real-time polymerase chain reaction (PCR) data. Neurosci Lett 2003, 339, 62-66, doi: 10.1016/s0304-3940(02)01423-4.

27. Rostam H.M., Reynolds P.M., Alexander M.R., Gadegaard N., Ghaemmaghami A.M.: Image based Machine Learning for identification of macrophage subsets. Sci Rep 2017, 7, 3521, doi: 10.1038/s41598-017-03780-z.

28. Schraml B.U., Reis e Sousa C.: Defining dendritic cells. Curr Opin Immunol 2015, 32, 13-20, doi: 10.1016/j.coi.2014.11.001.

29. Tao Z., Yang H., Jia D., Wan L., Cheng J., Lu X.: Molecular cloning, recombinant expression and characterization of GMCSF from the rhesus monkey, Macaca mulatta. Dev Comp Immunol 2013, 40, 69-77, doi: 10.1016/j.dci.2013.01.006.

30. Tran A.-M., Nguyen T.-T., Nguyen C.-T., Huynh-Thi X.-M., Nguyen C.-T., Trinh M.-T., Tran L.-T., Cartwright S.P., Bill R.M., Tran-Van H.: Pichia pastoris versus Saccharomyces cerevisiae: a case study on the recombinant production of human granulocyte-macrophage colony-stimulating factor. BMC Res Notes 2017, 10, doi: 10.1186/s13104-017-2471-6.

31. Wagner B., Perkins G., Babasyan S., Freer H., Keggan A., Goodman L.B., Glaser A., Torsteinsdóttir S., Svansson V., Björnsdóttir S.: Neonatal immunization with a single IL-4/antigen dose induces increased antibody responses after challenge infection with equine herpesvirus type 1 (EHV-1) at weanling age. PLoS One 2017, 12, doi: 10.1371/journal.pone.0169072. 